\title{
AN AXIOMATIC BASIS FOR PLANE GEOMETRY*
}

\author{
BY \\ STEWART S. CAIRNS
}

1. The axioms. The fourth appendix of Hilbert's Grundlagen der Geometrie $\dagger$ is devoted to the foundation of plane geometry on three axioms pertaining to transformations of the plane into itself. The object of the present paper is to attain the same end by quicker and simpler means. The simplifications are made possible by using orientation-reversing transformations $\ddagger$ and changing Hilbert's second and third axioms.

The $(x, y)$-plane will mean the set of all distinct ordered pairs of real numbers. The terms of analytic geometry, to which no geometric content need be given, will be used, modified by the prefix $(x, y)$ where ambiguity might arise. Thus we shall refer to $(x, y)$-distance, $(x, y)$-lines, and so on.

The general plane, $p$, will be any set of objects, called points, which can be put in one-to-one correspondence with the points of the $(x, y)$-plane. For convenience, we shall speak of the points of $p$ as if they were identical with their images under such a correspondence.

The following axioms pertain to a set, $T$, of continuous $\S$ one-to-one transformations of $p$ into itself. A transformation of the set which leaves two distinct points fixed and reverses orientation will be called a reflection.

Ахіом 1. The transformations $T$ form a group.

Axiom 2. If $A$ and $B$ are two points of $p, T$ contains a reflection leaving $A$ and $B$ fixed.

Aхгом 3. Let $T_{A}$ denote the subset of $T$ containing all the transformations thereof which leave $A$ fixed. If $T_{A}$ contains transformations carrying pairs of points arbitrarily near a given pair of points $(B, C)$ into an arbitrarily small neighborhood of a pair $(D, E)$, then $T_{A}$ contains a transformation carrying $(B$, $C)$ into $(D, E)$.

2. The curve $\gamma$. Our first object is to establisk the following theorem, which, like Lemma 1 below, is similar to a result employed by Hilbert (loc. cit.).

* Presented to the Society, September 9, 1930; received by the editors July 10, 1932. This paper was partly written while the writer was studying under Professor B. von Kerékjártó at Szeged University as a Travelling Fellow from Harvard University.

$\dagger$ D. Hilbert, Grundlagen der Geometrie, 1930, pp. 178-230.

$\ddagger$ Suggested by Hilbert, loc. cit., p. 182.

$\S$ That is, continuous in terms of $(x, y)$-distance. 
THEOREM 1. Every neighborhood of A contains a simple closed curve, $\gamma$, enclosing $A$ and preserved by each of the transformations $T_{A}$.

We shall assume the Jordan separation theorem and the following converse thereof:

(A) A locally connected ${ }^{*}$ set of points which divides the $(x, y)$-plane into two regions, one of them finite, and forms their common boundary, is a simple closed curve. $\dagger$

Lemma 1. For any given positive $\epsilon$, there is a neighborhood, $N$, of $A$ on $p$, no point of which is carried to a distance $\epsilon$ from $A$ by any of the transformations $T_{A}$ (Axiom 3).

Otherwise, let $P_{i}(i=1,2, \cdots)$ be a point within distance $\epsilon / 2^{i}$ of $A$, whose image, $Q_{i}$, under one of the transformations $T_{A}$ is at distance $\epsilon$ from $A$. Then $T_{A}$ contains transformations carrying points arbitrarily near $A$ into an arbitrarily small neighborhood of any cluster point, $Q$, of $\left(Q_{1}, Q_{2}, \cdots\right)$. Therefore, by Axiom 3, $T_{A}$ contains a transformation carrying $A$ into $Q$. But this is impossible, for the transformantions $T_{A}$ all leave $A$ fixed.

Lemma 2. Let $c$ be any simple closed curve on $N$ enclosing $A$. The set, $\Gamma$, of all points into which points on c are carried by the transformations $T_{A}$ is closed. The transformations $T_{A}$ all preserve $\Gamma$.

Any cluster point, $P$, of $\Gamma$ is limit of some series $\left(P_{1}, P_{2}, \cdots\right)$ on $\Gamma$. By definition of $\Gamma$, one of the transformations $T_{A}$ carries a point $Q_{i}(i=1,2, \cdots)$ on $c$ into $P_{i}$. Hence, if $Q$ is a cluster point, necessarily on $c$, of $\left(Q_{1}, Q_{2}, \cdots\right)$, $T_{A}$ (see Axiom 3) contains transformations carrying points arbitrarily near $Q$ into an arbitrary neighborhood of $P$. Therefore (Axiom 3), $T_{A}$ contains a transformation carrying $Q$ into $P$. Hence $P$ is on $\Gamma$, and $\Gamma$ is closed.

Consider the image, $P^{\prime}$, of any point, $P$, on $\Gamma$ under any transformation, $T_{1}$, of the set $T_{A}$. Let $T_{0}$ be one of the transformations $T_{A}$ carrying some point, $Q$, on $c$ into $P$. Then $T_{0} T_{1}$ carries $Q$ into $P^{\prime}$. Since $T_{0} T_{1}$ leaves $A$ fixed and belongs to $T$ (Axiom 1), it belongs also to $T_{A}$. Therefore $P^{\prime}$ is on $\Gamma$. This completes the proof.

* A point set, $S$, is said to be locally connected if, for any $\epsilon>0$ and any point $P$, of $S$, there exists a positive distance, $\delta$, such that all points of $S$ within distance $\delta$ of $P$ are connected with $P$ by a subset of $S$ entirely within distance $\epsilon$ of $P$.

$\dagger$ Essentially in this form, the theorem is given by J. R. Kline, these Transactions, vol. 21 (1920), p. 452. It is a ready consequence of Hahn's characterisation of continuous curves, Jahresbericht der Deutschen Mathematiker Vereinigung, vol. 23 (1914), p. 318, together with a theorem by R. L. Moore, Bulletin of the American Mathematical Society, vol. 23 (1917), p. 233, that any two points of a continuous curve, $S$, can be joined on $S$ by a simple Jordan arc. 
LEMмA 3. The complement of $\Gamma$ on the $(x, y)$-plane contains just one unlimited region, $R$. The boundary, $\gamma$, of $R$ divides the $(x, y)$-plane into two regions, $R$ and $R_{0}$, and forms their common boundary.

The first part of the lemma follows from Lemmas 1 and 2. It also follows from these lemmas that $\gamma$ is on $\Gamma$. Let $P$ denote any point neither in $R$ nor on $\gamma$, and $k$ a simple arc through $P$ with just its end points, $P_{1}$ and $P_{2}$, on $\gamma$. Some transformation, $T_{j}(j=1,2)$, of the set $T_{A}$ carries a point, $Q_{j}$, of $c$ into $P_{j}$ (Lemma 2). A simple arc inside $c$ joining $A$ to $Q_{j}$ is carried by $T_{j}$ into an arc, $k_{j}$, joining $A$ to $P_{j}$ but not meeting either $R$ or $\gamma$. Because $R$ is connected, $\left(k+k_{1}+k_{2}\right)$ cannot enclose any point of either $R$ or its boundary, $\gamma$. Therefore $\gamma$ cannot separate $P$ from $A$. Hence all points neither in $R$ nor on $\gamma$ are in a single region, $R_{0}$. By such a curve as $k_{1}$, any point on $\gamma$ can be joined to $A$ inside $R_{0}$. Therefore, $\gamma$ is the common boundary of $R_{0}$ and $R_{1}$.

\section{LEMMA 4. The boundary $\gamma$ is locally connected.}

Suppose that at some point, $X, \gamma$ is not locally connected. Then a positive number, $d$, exists, so small that every neighborhood of $X$ contains points on $\gamma$ not connected with $X$ by any continuum on $\gamma$ entirely within distance $d$ of $X$. Let $P_{i}(i=1,2, \cdots)$ be one such point within distance $d / 2^{i}$ of $X$. Let $C$ denote the $(x, y)$-circle of radius $d$ about $X$ and $K_{i}$ the set of all points connected with $P_{i}$ on $\gamma$ inside $C$. Then, if $K_{i}$ and $K_{j}$ have a point in common, they coincide. It may readily be seen that $K_{i}$ contains all its cluster points inside $C$. Therefore, at most a finite number of the $K$ 's can coincide with any one of them. Otherwise, an infinite subset of $\left(P_{1}, P_{2}, \cdots\right)$ would belong to one of the $K$ 's, which would therefore contain $X$ and join it in $C$ to certain of the $P$ 's. Hence, with no loss of generality, we may assume that the $K$ 's are all distinct.*

Let $C_{1}$ be the circle of radius $d / 2$ about $X$, and $K_{i}^{\prime}(i=1,2, \cdots)$ a closed connected subset of $K_{i}$ joining $P_{i}$ to $C_{1}$ but containing no points outside $C_{1}$. Let $C_{2}$ be the circle of radius $d / 4$ about $X$. Without loss of generality, we assume* that $K_{i}^{\prime}$ contains a point, $Q_{i}$, on $C_{1}$ and a point, $S_{i}$, on $C_{2}$ such that $\left(Q_{1}, Q_{2}, \cdots\right)$ converges to a limit, $Q$, monotonically on the arc $Q_{1} Q_{2} Q$, and $\left(S_{1}, S_{2}, \cdots\right)$ converges to a limit, $S$, monotonically on the $\operatorname{arc} S_{1} S_{2} S$. Consider, for any $i>1$, a simple closed curve made up of two arcs, $k_{1}$ and $k_{2}$, joining $A$ to $S_{i}$ inside $R_{0}$ (Lemma 3, proof). Suppose $k_{1}$ meets the arc $\alpha_{i} \equiv Q_{i-1} Q_{i} Q_{i+1}$ on $C_{1}$, but not the broken line $\beta_{i} \equiv P_{i-1} P_{i} P_{i+1}$, whereas $k_{2}$ meets $\beta_{i}$ but not $\alpha_{i}$. Then $\left(k_{1}+k_{2}\right)$ separates $S_{i-1}$ from $S_{i+1}$. For, let a simple

* To avoid excessive notation, we assume for the $K$ 's several properties enjoyed by some infinite subset thereof. 
closed curve, $\gamma_{0}$, be formed by adding to $\alpha_{i}$ and $\beta_{i}$ a pair of arcs joining $Q_{i-1}$ to $P_{i-1}$ and $Q_{i+1}$ to $P_{i+1}$, respectively. Let these latter curves pass through $S_{i-1}$ and $S_{i+1}$, respectively, and lie so near to $K_{i-1}^{\prime}$ and $K_{i+1}^{\prime}$ that $\gamma_{0}$ encloses $S_{i}$ and is met by $k_{1}$ only on $\alpha_{i}$ and by $k_{2}$ only on $\beta_{i}$. Then $\left(k_{1}+k_{2}\right)$ clearly contains just one arc inside $\gamma_{0}$ separating $S_{i-1}$ from $S_{i+1}$. Therefore $S_{i-1}$ and $S_{i+1}$ are separated by the closed curve $\left(k_{1}+k_{2}\right)$. But this is impossible, for no curve in $R_{0}$ can enclose points of $R$ (Lemma 3). Therefore, if $c_{i}$ is a simple closed curve in $R_{0}$ through $A$ and $S_{i}$, then $\alpha_{i}$ (or $\beta_{i}$ ) meets both of the arcs into which $c_{i}$ is divided by $A$ and $S_{i}$.

Now $S_{i}(i=1,2, \cdots)$, being on $\Gamma$ (Lemmas 2,3$)$, is image, under a transformation, $T_{i}$, of the set $T_{A}$, of some point, $E_{i}$, on the curve $c$ of Lemma 2. Let $c^{\prime}$ be a simple closed curve through $A$ which contains no points outside $c$ but has in common with $c$ an arc through a cluster point, $E$, of $\left(E_{1}, E_{2}, \cdots\right)$. With no loss of generality, we assume* that all the $E^{\prime}$ s lie on $c^{\prime}$ and that $\left(E_{1}, E_{2}, \cdots\right)$ converges to $E$. Then $T_{i}$ carries $c^{\prime}$ into a simple closed curve, $c_{i}$, to which the conclusion of the preceding paragraph applies. $\dagger$ We shall treat only the case where both the $\operatorname{arcs} A S_{i}(i=1,2, \ldots)$ on $c_{i}$ meet $\alpha_{i} . \ddagger$ In this case, $c^{\prime}$ passes through two points, $E_{i}^{\prime}$ and $E_{i}^{\prime \prime}$, separated on $c^{\prime}$ by $\left(A, E_{i}\right)$, where the images of $\left(E_{i}^{\prime}, E_{i}^{\prime \prime}\right)$ under $T_{i}$ are on $\alpha_{i}$. Without loss of generality, we assume* that $\left(E_{1}^{\prime}, E_{2}^{\prime}, \cdots\right)$ and $\left(E_{1}^{\prime \prime}, E_{2}^{\prime \prime}, \cdots\right)$ converge to a pair of points, $E^{\prime}$ and $E^{\prime \prime}$, respectively. Now, by definition, $\alpha_{i}$, for $i$ large enough, is in an arbitrarily small given neighborhood of $Q$. Hence, since $T_{i}$ carries $\left(E_{i}^{\prime}, E_{i}^{\prime \prime}\right)$ onto $\alpha_{i}, T_{A}$ contains a transformation (Axiom 3) carrying $\left(E^{\prime}, E^{\prime \prime}\right)$ into $Q$. Hence $E^{\prime} \equiv E^{\prime \prime}$. Since $A$ and $E_{i}$ separate $E_{i}^{\prime}$ and $E_{i}^{\prime \prime}$ on $c^{\prime}, E^{\prime}$ and $E^{\prime \prime}$ can coincide only at $A$ or at $E$. But $A$ cannot go into $Q$ under any of the transformations $T_{A}$. Hence $E^{\prime} \equiv E^{\prime \prime} \equiv E$. Then, for $i$ large enough, $T_{i}$ carries a pair of points $\left(E_{i}, E_{i}^{\prime}\right)$ arbitrarily near $E$ into an arbitrary neighborhood of the pair $(S, Q)$. Therefore (Axiom 3) $T_{A}$ contains a transformation carrying $E^{\prime}$ into $(S, Q)$. This contradicts the one-to-one-ness of the transformations and establishes the lemma.

Theorem 1 above is an immediate consequence of (A) together with Lemmas 2,3 , and 4 .

3. Lines and reflections. The set of all fixed points under a reflection (see $\$ 1$ ) will be called a line.

* See footnote on p. 236.

$\dagger$ To show this, $c^{\prime}$ may be slightly deformed, if necessary, so that its image meets $\gamma$ only at $S_{i}$.

$\ddagger$ The method applies equally well if both arcs meet $\beta_{i}$. We need only replace $\alpha$ by $\beta$ and $Q$ by $P$. In assuming that the arc $A S_{i}$ meets $\alpha_{i}$ (or $\beta_{i}$ ) for all values $i$, we employ the convention stated in the footnote on p. 236. 
LEMMA 1. An orientation-preserving transformation, $\tau$, of the group $T$ which preserves a simple closed curve, $\gamma$, and leaves one point of $\gamma$ fixed, leaves every point of $\gamma$ fixed.*

If $A$ denote the known fixed point on $\gamma$, then $\tau$ and all its powers belong to the set $T_{A}$ (Axiom 3). Let $P_{i}$ be the image of $P_{0}$ under the $i$ th power of $\tau$. Ascribing a positive sense to $\gamma$, consider the $\operatorname{arc} \overrightarrow{A P}$. If it passes through $P_{1}$, then the arc $\overrightarrow{A P}_{1}$, being the image under $\tau$ of $\overrightarrow{A P}_{0}$, passes through $P_{2}$; and, in general, $\overrightarrow{A P}_{i}(i=2,3, \cdots)$ passes through $P_{i+1}$, but not $P_{i-1}$. On the other hand, if $\overrightarrow{P_{0} A}$ on $\gamma$ passes through $P_{1}$, then $\overrightarrow{P_{i} A}$ contains $P_{i+1}$ but not $P_{i-1}$. Thus, in either case, $\left(P_{1}, P_{2}, \cdots\right)$ is a monotonic series on the curve $\gamma$. If $P$ is its limit, then, for $i$ sufficiently large, the two points $\left(P_{i}, P_{i+1}\right)$ are in an arbitrary preassigned neighborhood of $P$. But these two points are images, under $\tau^{i}$, of $\left(P_{0}, P_{1}\right)$. Therefore (Axiom 3) some transformation of the set $T_{A}$ carries both $P_{0}$ and $P_{1}$ into $P$. This implies that $P_{0}$ and $P_{1}$ coincide, and hence that every point of $C$ is fixed under $\tau$.

CoRollary. The transformation $\tau$ is the identity.

First, since $\tau$ is continuous, the set, $S$, of its fixed points is closed with respect to $p$. Suppose $S$ does not coincide with $p$, and consider the largest connected subset, $S_{1}$, of $S$ which contains $C$. Let $k$ be a simple curve joining a point, $Q$, of $(p-S)$ to a point, $B$, of $S_{1}$, but not containing any point of $\left(S_{1}-B\right)$. Let $\gamma^{\prime}$ be a simple closed curve about $B$ which meets both $k$ and $S_{1}$ and which is carried into itself by $\tau$ (Theorem 1). Since $\gamma^{\prime}$ meets $S_{1}$, all its points are fixed under $\tau$ and hence belong to $S_{1}$. This contradicts the definition of $k$ and thus establishes the corollary.

Lemma 2. A simple closed curve which is preserved by a reflection, $\rho$, is met in just two points by the line which $\rho$ defines.

For, an orientation-reversing transformation which preserves a simple closed curve leaves just two points on the curve fixed.

LEMMa 3. The identity is the only orientation-preserving transformation of the group $T$ which leaves every point of a line, $L$, fixed.

This follows from the preceding results of this section applied to the curve $\gamma$ of Theorem 1, where $A$ is on $L$.

THEOREM 2. A reflection, $\rho$, is involutory. No two different reflections define the same line, $L$.

\footnotetext{
* The proof is patterned after one by Hilbert, loc. cit., pp. 204, 205.
} 
By Lemma 3, $\rho \rho$ is the identity. Also, if $\rho$ and $\rho^{\prime}$ both define $L, \rho \rho^{\prime}$ is the identity. Therefore $\rho=\rho^{\prime}$.

Corollary. A reflection preserves the set of all lines.

Let $L_{1}$ be any line and $L_{2}$ its image under an arbitrary reflection, $\rho$. Let $\rho_{1}$ be the reflection defining $L_{1}$. Then, since reflections are involutory, $\rho \rho_{1} \rho$ leaves just the points on $L_{2}$ fixed and reverses orientation. Therefore $\rho \rho_{1} \rho$ is a reflection and $L_{2}$ a line.

4. Properties of the line. (A) Let $\rho$ be a reflection and $\left(P_{1}, P_{2}\right)$ a pair of points interchanged by $\rho$ (Theorem 2). Then any simple Jordan arc, $k$, which joins $P_{1}$ and $P_{2}$ meets the line, $L$, defined by $\rho$.

Since $\rho$ is involutory (Theorem 2), it interchanges $k$ with its image, $k^{\prime}$. As a point, $Q$, traces $k$ from $P_{1}$ to $P_{2}$, its image, $Q^{\prime}$, under $\rho$ traces $k^{\prime}$ from $P_{2}$ to $P_{1}$. As the $\operatorname{arc} P_{1} Q$ on $k$ increases, $Q$ reaches a first position, $Q_{1}$, which is on the image, under $\rho$, of the $\operatorname{arc} P_{1} Q$, end points included. Let $Q_{2}$ be the image of $Q_{1}$. If $Q_{1}$ is not on $L$, the $\operatorname{arcs} Q_{1} Q_{2}$ on $k$ and $k^{\prime}$, respectively, have only their end points in common. Since $\rho$ interchanges these arcs and reverses orientation, it must leave $Q_{1}$ and $Q_{2}$ fixed. Therefore $Q_{1}\left(\equiv Q_{2}\right)$ is on $L$.

Lemma. A line, $L$, is locally connected.

Under a contrary assumption, let $A$ denote a point at which $L$ is not locally connected; and let $c$ denote an $(x, y)$-circle about $A$, so small that every neighborhood of $A$ contains points of $L$ not connected with $A$ on $L$ inside $c$. In particular, consider the neighborhood $N$ of Lemma 1 in $\S 2$, where $\epsilon$ is the radius of $c$. Let $k$ be a simple arc in $N$, with only its end points on $L$, joining two points not connected on $L$ inside $c$. Then, by (A), $k$ and its image, $k^{\prime}$, under the reflection defining $L$ are distinct and are separated by the points of $L$ inside $\left(k+k^{\prime}\right)$. Hence these points of $L$ join the end points of $k$; but this is contradictory, since, by definition of $N,\left(k+k^{\prime}\right)$ is entirely inside $c$.

(B) If $c$ is a simple arc with just its end points on a given line, $L$, then $c$ and its image, $\gamma$, under the reflection, $\rho$, defining $L$, form a simple closed curve. The points of $L$ inside $(c+\gamma)$ constitute a simple Jordan arc joining the common end points of $c$ and $\gamma$.

From (A), $(c+\gamma)$ is a simple closed curve. Let $R$ be the set of all points which can be connected with $c$ by a simple arc inside $(c+\gamma)$ containing no points of $L$. Let $\lambda$ denote the set of points common to $L$ and the boundary of $R$. The image, $R^{\prime}$, of $R$ under $\rho$ obviously consists of all the points which can be connected with $\gamma$ inside $(c+\gamma)$ without meeting $L$. By (A), $R$ and $R^{\prime}$ are distinct. Therefore $\lambda$ separates $c$ from $\gamma$ and connects their common end points. Also, $\left(R+\lambda+R^{\prime}\right)$ contains all points inside $(c+\gamma)$, for otherwise some 
point would belong to a finite region bounded solely by points on $L$, and this region would go into itself under $\rho$ in contradiction with (A). Now $(c+\lambda)$ divides the $(x, y)$-plane into two regions, the finite region $R$ and the region consisting of $R^{\prime}$ plus $\gamma$ plus the exterior of $(c+\gamma)$; and $(c+\lambda)$ is the common boundary of these two regions. Hence, using the above Lemma and §2(A), $(c+\lambda)$ is a simple closed curve, and $\lambda$ is an arc thereof.

(C) A line, $L$, is homeomorphic with the $x$-axis.

Let $c_{1}$ be a simple arc with just its end points $\left(A_{1}, B_{1}\right)$ on $L$, and let $Q$ be a point of $c_{1}$. Then $c_{1}$ plus its image, $\gamma_{1}$, under the reflection, $\rho$, defining $L$ cuts from $L$ a Jordan arc, $\lambda_{1}$ (see $(B)$ ). Let $\lambda_{1}$ be put in continuous one-to-one correspondence with the segment $-1 \leqq x \leqq 1$ on the $x$-axis, $A_{1}$ corresponding to -1 and $B_{1}$ to +1 .

Proceeding inductively, for $i=1,2, \cdots$, let $c_{i+1}$ be a simple arc which passes through $Q$, has only its end points on $L$, and lies outside $\left(c_{i}+\gamma_{i}\right)$. Further, let $c_{i+1}$ pass through no point within some positive preassigned distance, $d$, of the points $A_{i}$ and $B_{i}$. The curve $c_{i+1}$ plus its image, $\gamma_{i+1}$, under $\rho$ cuts from $L$ a simple arc, $\lambda_{i+1}$, containing $\lambda_{i}$. Let $\lambda_{i+1}$ be put in continuous one-to-one correspondence with the interval $-(i+1) \leqq x \leqq(i+1)$ on the $x$-axis in such a way as to preserve the correspondence of $\lambda_{i}$ with the interval $-i \leqq x \leqq i$. Let $\left(A_{i+1}, B_{i+1}\right)$ be the end points of $\lambda_{i+1}$ which correspond to $-(i+1)$ and $(i+1)$, respectively. By the last condition imposed on $c_{i+1}$, neither of the series $\left(A_{1}, A_{2}, \cdots\right)$ and $\left(B_{1}, B_{2}, \cdots\right)$ has a cluster point on $p$. Hence the above inductive process leads to a continuous one-to-one correspondence between the $x$-axis and a portion, $L^{\prime}$, of $L$ where $L^{\prime}$ divides $p$ into two parts. Now the set of all points each inside at least one of the curves $\left(c_{i}+\gamma_{i}\right)(i=1,2, \cdots)$ is a neighborhood of $L^{\prime}$ free from other points of $L$. Hence $L^{\prime}$ and $\left(L-L^{\prime}\right)$ are distinct. If the set $\left(L-L^{\prime}\right)$ is not vacuous, let $c^{\prime}$ be an arc with just its end points on $L$, one end point being on $L^{\prime}$ and one on $\left(L-L^{\prime}\right)$. By (B), the end points of $c^{\prime}$ are joined on $L$ by a simple arc. This contradiction establishes that $L^{\prime} \equiv L$.

(D) If two lines, $L$ and $L^{\prime}$, through any point, $A$, have any other point, $B$, of the neighborhood $N(\$ 2$, Lemma 1$)$ in common, they coincide.

Let $\gamma_{0}$ be a curve about $A$ satisfying Theorem 1 and not enclosing $B$. Suppose $\gamma_{0}$ passes through no common point of $L$ and $L^{\prime}$. As the $\operatorname{arc} A B$ on $L$ is traced from $A$, let $B^{\prime}$ be the first point reached outside $\gamma_{0}$ and on $L^{\prime}$. Then $B^{\prime}$ is joined to $\gamma_{0}$ by two distinct arcs, $k$ and $k^{\prime}$, on $L$ and $L^{\prime}$, respectively. Let $c$ be the simple closed curve about $A$ formed of $k, k^{\prime}$ and an arc on $\gamma_{0}$. Using this for the curve $c$ of Lemmas 2, 3, etc., in $\$ 2$, we are led to a curve, $\gamma$, satisfying Theorem 1 . This curve passes through $B^{\prime}$. Suppose it 
does not. Then $A$ and $B^{\prime}$ are both inside $\gamma$; and, since $L$ and $L^{\prime}$ meet $\gamma$ each in just two points, the $\operatorname{arcs} A B^{\prime}$ on $L$ and $L^{\prime}$ respectively; are both inside $\gamma$. Hence $c$ and $\gamma$ meet only on $\gamma_{0}$. But then ${ }^{*} \gamma=\gamma_{0}$, which is impossible. Since, therefore, $\gamma$ passes through the common point $B^{\prime}$ of $L$ and $L^{\prime}$, the product of the reflections defining $L$ and $L^{\prime}$ is the identity ( $\$ 3$, Lemma 1 and Corollary), and $L \equiv L^{\prime}$ (Theorem 2).

5. Further properties. The connection with euclidean plane geometry. The remaining developments prepare for a complete deduction of euclidean plane geometry.

Lemma 1. Let $\left(L_{0}, L_{1}\right)$ be two different lines through any point, $A$, and let $\left(A_{j}, B_{j}\right)(j=0,1)$ be the two points $(\$ 3$, Lemma 2$)$ in which $L_{j}$ meets the curve $\gamma$ of Theorem 1. Then the points $\left(A_{0}, B_{0}\right)$ separate $A_{1}$ from $B_{1}$ on $\gamma$.

Suppose the contrary, and let $\gamma^{\prime}$ denote the $\operatorname{arc} A_{0} B_{0}$ on $\gamma$ containing $A_{1}$ and $B_{1}$. Under the reflection defining $L_{1}, L_{0}$ goes into a line, $L_{2}$, which meets $\gamma$ in the images $\left(A_{2}, B_{2}\right)$ of $\left(A_{0}, B_{0}\right)$, and, by $\$ 4(\mathrm{~A})$, both $A_{2}$ and $B_{2}$ must be on the $\operatorname{arc} A_{1} B_{1}$ of $\gamma^{\prime}$. Proceeding inductively for $i=1,2, \cdots$, let $L_{2 i+1}$ and $L_{2 i+2}$ be the images of $L_{1}$ and $L_{0}$, respectively, under the reflection, $\rho_{2 i}$, defining $L_{2 i}$. Let $\left(A_{2 i+1}, A_{2 i+2}\right)$ be the images under $\rho_{2 i}$ of $\left(A_{1}, A_{0}\right)$ and $\left(B_{2 i+1}, B_{2 i+2}\right)$ the images of $\left(B_{1}, B_{0}\right)$. Then the $\operatorname{arc} A_{j} B_{j}$ on $\gamma^{\prime}$ contains $A_{j+1}$ and $B_{j+1}$. Thus the series $\left(A_{1}, A_{2}, \cdots\right)$ converges monotonically on $\gamma^{\prime}$ to a limit, $X$. Hence, for $i$ large enough, $A_{2 i+1}$ and $A_{2 i+2}$ are within an arbitrary given distance of $X$. By Axiom 3, since $\rho_{2 i}$ belongs to $T_{A}, T_{A}$ contains a transformation carrying $\left(A_{0}, A_{1}\right)$ into $X$. But our transformations are all one-to-one. This contradiction establishes the desired result.

Let $(A, P)$ denote any pair of distinct points on the plane $p$. The set of all images of $P$ under reflections leaving $A$ fixed will be called a circle, with $A$ as center.

Theorem 3. A circle, $K$, is a simple closed curve.

(I) Suppose the point $P$ of the above definition is in a neighborhood, $N$, of $A$ satisfying Lemma 1 in $\S 2$, so that $K$ is in a finite region of the $(x, y)$ plane. Each point, $P^{\prime}$, on $K$ is image of $P$ under just one reflection leaving $A$ fixed. For suppose there were two such reflections $\left(\rho_{1}, \rho_{2}\right)$ carrying $P$ into $P^{\prime}$. Let $\rho$ be the reflection defining the line $A P^{\prime}$. Then $\rho_{1} \rho \rho_{2}$ and $\rho_{1} \rho \rho_{1}$ are both reflections leaving $A$ and $P$ fixed. Hence they both define the unique line $(\$ 4(D))$ through $A$ and $P$. Therefore $\rho_{1} \rho \rho_{2} \equiv \rho_{1} \rho \rho_{1}$ (Theorem 2), or $\rho_{1} \equiv \rho_{2}$.

Using the notation of Lemma 1 above, let $\gamma^{\prime}$ be one of the two $\operatorname{arcs} A_{0} B_{0}$ on $\gamma$. As a point, $Q$, traces $\gamma^{\prime}$ from $A_{0}$ to $B_{0}$, the line through $A$ and $Q$ adopts

* Since $\gamma$ is the set of images under $T_{A}$ of points common to $c$ and $\gamma$ (Theorem 1, and Lemmas 2,3 in $\$ 2$ ), and $T_{A}$ preserves $\gamma_{0}$. 
the position of every line through $A$ once and only once (Lemma 1), except that the same line is obtained for $Q \equiv A_{0}$ as for $Q \equiv B_{0}$. To eliminate the exception, regard $A_{0}$ and $B_{0}$ as identical, so that $Q$ traces essentially a simple closed curve. Then the image, $P^{\prime}$, of $P$ under the reflection defining the line $A Q$ adopts every position on $K$ once and only once as $Q$ traces $\gamma^{\prime}$. This affords a one-to-one correspondence between the points on $K$ and those on $\gamma^{\prime}$, the point $A_{0}\left(\equiv B_{0}\right)$ included. It remains only to show the continuity of this correspondence. Let $Q$ be any point on $\gamma^{\prime}$ and $\left(Q_{1}, Q_{2}, \cdots\right)$ a series of points converging to $Q$. Let $P_{i}(i=1,2, \cdots)$ be the point on $K$ corresponding to $Q_{i}$ and let $P^{0}$ be any cluster point of the $P$ 's. Then there are transformations leaving $A$ fixed, carrying points arbitrarily near $Q$ into themselves and carrying $P$ into an arbitrary neighborhood of $P^{0}$. Hence (Axiom 3) there is a transformation leaving $A$ and $Q$ fixed and carrying $P$ into $P^{0}$. This must be the reflection in the line $A Q(\$ 4(D) ; \S 3$, Lemma 3 and Theorem 2). Therefore $P^{0}$ is the point corresponding to $Q$. This completes the argument for this special case.

(II) Suppose the theorem false. Then, by (I), as some line, $L$, is traced from $A$ in one sense or the other, a point, $P$, is reached which is either the last position for which $K$ is a simple closed curve, or the first for which it is not. In the first case, by a proof like that of Lemma $1 \mathrm{in} \S 2, K$ has a neighborhood consisting of points which remain within distance $\epsilon$ of $K$ under all transformations of the set* $T_{A}$. Since this neighborhood contains $P$ it follows from (I) that $P$ cannot be the last point for which $K$ is a simple closed curve.

We deal with the second case by showing that if every internal point of the arc $A P$ on $L$ generates a circle which is a simple closed curve, then the circle, $K$, generated by $P$ is also a simple closed curve. Let it first be noted that no two different lines $\left(L_{1}, L_{2}\right)$ through $A$ can meet at a point, $Q$, on $K$. If they did, then, by $\S 4(\mathrm{D})$, the $\operatorname{arcs} A Q$ on $L_{1}$ and $L_{2}$ would be distinct and hence form a simple closed curve, $c$. But then every line determined by $A$ and a point inside $c$ would clearly pass through $Q$. By reflections in $L_{1}$ and $L_{2}$ one can show that other lines through $A$ pass through $Q$; indeed, one can show that all lines through $A$ pass through $Q$, from which it is easy to deduce a contradiction. Now, for any $\epsilon>0$, there exists a neighborhood, $N_{\epsilon}$, of $P$, such that no image of $P$ under the reflection defining a line through a point of $N_{\epsilon}$ is at distance greater than $\epsilon$ from $P$. This may be established by an argument similar to that of Lemma 1 in $\$ 2$. Consider the correspondence employed in (I) above. We have seen that it is one-to-one even in the present case. We can establish its continuity by an argument like that in (I) applied

* The proof of Corollary 1 below shows that these transformations preserve $K$. 
to images of $P$ in $N_{\epsilon}$, and, similarly, in a neighborhood of any point on $K$. Hence $K$ is a simple closed curve.

CoROLlaRy 1. A circle with center at $A$ is preserved by all the transformations $T_{A}($ Axiom 3).

Let $\tau$ be a transformation of the set $T_{A}$. Let $P^{\prime \prime}$ be the image under $\tau$ of any point $P^{\prime}$ on $K$, and let $\rho$ be the reflection which leaves $A$ fixed and interchanges $\left(P, P^{\prime}\right)$. Then, if $\rho^{\prime}$ is the reflection defining the line $A P^{\prime}, \rho \tau$ and $\rho \rho^{\prime} \tau$ both carry $P$ into $P^{\prime \prime}$ and leave $A$ fixed. The one which reverses orientation is a reflection, for it preserves the curve $\gamma$ of Theorem 1 and therefore leaves two of its points fixed. Hence $P^{\prime \prime}$ is on $K$.

Corollary 2. A circle is met in just two points by a line through its center. (See §3, Lemma 2.)

Corollary 3. Any two points $\left(P_{1}, P_{2}\right)$ on a circle are interchanged by some reflection leaving the center fixed.

Let $\rho_{j}$ be the reflection leaving the center, $A$, fixed and carrying $P$ into $P_{j}(j=1,2)$. If $\rho$ define the line through $A$ and $P$, the product $\rho_{1} \rho \rho_{2}$ reverses orientation, carries $P_{1}$ into $P_{2}$ and preserves the circle. It is therefore a reflection, for it leaves two points on the circle fixed. Hence (Theorem 2) it interchanges $P_{1}$ and $P_{2}$.

THEOREM 4. One and only one line passes through any two distinct points of the plane $p$.

If two lines through a point, $A$, have a second point, $P$, in common, their defining reflections preserve the circle through $P$ with center at $A$ and leave $P$ fixed. The product of these reflections is therefore the identity and the lines coincide $(\$ 3)$.

Corollary 1. For any two points, $A$ and $B$, on the plane $p$, there exists a reflection interchanging them.

Let $P$ be a common point of the two circles, centers at $A$ and $B$, respectively, passing through $B$ and $A$, respectively. Let $\rho_{1}$ be the reflection interchanging $A$ and $P$ and leaving $B$ fixed (Theorem 3, Corollary 3) and $\rho_{2}$ the reflection interchanging $B$ and $P$ and leaving $A$ fixed. Then $\rho_{1} \rho_{2} \rho_{1}$ reverses orientation, leaves $P$ fixed, and carries $A$ into $B$. Since $\rho_{1}$ and $\rho_{2}$ are involutory (Theorem 2), $\rho_{1} \rho_{2} \rho_{1}$ is also involutory and therefore leaves more than one point fixed (see proof of $\$ 4(\mathrm{~A})$ ). Hence it is the required reflection.

Corollary 2. Let $L$ and $L^{\prime}$ be any two lines. Let $A$ be any point on $L$ and $B$ any point on $L^{\prime}$. Then some transformation in the group $T$ carries $L$ into $L^{\prime}$ in such a way that $A$ goes into $B$. 
The reflection, $\rho_{1}$, which interchanges $A$ and $B$ (Corollary 1) carries $L$ into a line, $L^{\prime \prime}$, through $B$ (Theorem 2 , Corollary). Some reflection, $\rho_{2}$, by Theorem 3, Corollary 3 , leaves $B$ fixed and interchanges any two points in which $L^{\prime}$ and $L^{\prime \prime}$, respectively, meet a circle, center at $B$. The product $\rho_{1} \rho_{2}$ satisfies the requirements of the present corollary.

There remain no difficulties in defining angles, distances, congruences, and proceeding with other geometrical developments, or else establishing the axioms in Chapter 1 of Hilbert's Grundlagen der Geometrie.

Two geometries rest on the above foundation: the euclidean if one assume that through a given point not on a given line, $L$, there is but one line which fails to meet $L$; the Bolyai-Lobatchewsky if one assume that there are two lines through the point separating the intersecting from the non-intersecting lines.

LEHIGH ÜNIVERSITY, Bethlehem, PA. 